ARTICLE

\title{
Completely non-fused electron acceptor with 3D-interpenetrated crystalline structure enables efficient and stable organic solar cell
}

Lijiao Ma ${ }^{1,2}$, Shaoqing Zhang (i] ${ }^{3 凶}$, Jincheng Zhu ${ }^{3}$, Jingwen Wang ${ }^{1,2}$, Junzhen Ren ${ }^{1}$, Jianqi Zhang ${ }^{4} \&$ Jianhui Hou (1) 1,2区

Non-fullerene acceptors (NFAs) based on non-fused conjugated structures have more potential to realize low-cost organic photovoltaic (OPV) cells. However, their power conversion efficiencies (PCEs) are much lower than those of the fused-ring NFAs. Herein, a new bithiophene-based non-fused core (TT-Pi) featuring good planarity as well as large steric hindrance was designed, based on which a completely non-fused NFA, A4T-16, was developed. The single-crystal result of A4T-16 reveals that a three-dimensional interpenetrating network can be formed due to the compact $\pi-\pi$ stacking between the adjacent end-capping groups. A high PCE of $15.2 \%$ is achieved based on PBDB-TF:A4T-16, which is the highest value for the cells based on the non-fused NFAs. Notably, the device retains $~ 84 \%$ of its initial PCE after $1300 \mathrm{~h}$ under the simulated AM $1.5 \mathrm{G}$ illumination $\left(100 \mathrm{~mW} \mathrm{~cm}^{-2}\right)$. Overall, this work provides insight into molecule design of the non-fused NFAs from the aspect of molecular geometry control.

\footnotetext{
${ }^{1}$ State Key Laboratory of Polymer Physics and Chemistry, Beijing National Laboratory for Molecular, Sciences CAS Research/Education Center for Excellence in Molecular Sciences, Institute of Chemistry Chinese Academy of Sciences, Beijing, People's Republic of China. ${ }^{2}$ University of Chinese Academy of Sciences, Beijing, People's Republic of China. ${ }^{3}$ School of Chemistry and Biology Engineering, University of Science and Technology Beijing, Beijing, People's Republic of China. ${ }^{4}$ CAS key laboratory of nanosystem and hierarchical fabrication, CAS Center for Excellence in Nanoscience, National Center for Nanoscience and

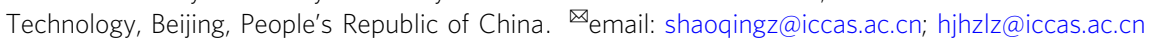


O rganic photovoltaic (OPV) cells have the outstanding advantage of fabricating large area and flexible devices through the low-cost solution coating techniques and hence attracted considerable attention in the past decades ${ }^{1-3}$. Benefiting from the development of various new OPV materials, especially the fused-ring non-fullerene acceptors (NFAs) with the so-called A-D-A structure, the power conversion efficiencies (PCEs) of OPV cells under the illumination of simulated solar light (AM $1.5 \mathrm{G}, 100 \mathrm{~mW} \mathrm{~cm}^{-2}$ ) have been significantly improved $^{4-10}$. In 2015, a new A-D-A-structured acceptor namely ITIC based on a seven fused-ring building block (DTIDT) was designed by Zhan et al., which demonstrated the possibility of utilizing NFA to realize high $\mathrm{PCE}^{4,5}$. Thereafter, many fused-ring building blocks were designed and applied in the molecular design of the NFAs ${ }^{11-15}$. In 2019, Zou et al. synthesized Y6, another new NFA featuring a more complex N-hetero-fused-ring building block, and realized even higher $\mathrm{PCE}^{8}$. Nowadays, although great progress in PCE has been made by employing these fused-ring-based NFAs ${ }^{16-20}$, the synthetic costs of the fused-ring blocks are too high to use on a relatively large scale for the future practical application ${ }^{21-23}$. There is still an urgent need to explore the new structural design of simple and effective molecules to meet the requirement of cost-effectiveness.

As the core units in the A-D-A-structured NFAs, the fusedring building blocks play a critical role because they have the following characteristics. On the one hand, the conjugated backbones in these building blocks should be planar and rigid, which is beneficial for $\pi$-electron delocalization and intramolecular charge transport. As these fused-ring building blocks, all contain a few sulfur-and/or nitrogen-heteroaromatic five-membered rings and thus are electron-rich ${ }^{4,8,17}$, a strong intramolecular charge transfer (ICT) effect can be formed when they conjugately link with the electron-deficient end-capping groups, which endows the flexibilities in modulating absorption spectra and molecular energy levels of the NFAs ${ }^{24-27}$. On the other hand, the bulky alkyls on the fused-ring building blocks not only guarantee good solubilities of the NFAs ${ }^{25,27,28}$ but also ensure the intermolecular $\pi-\pi$ stacking of the NFAs to mainly occur at the end-capping groups, because such a molecular packing mode is crucial for their electron-accepting capability $19,20,29-31$. Putting all of the above characteristics together, it is a big challenge to replace the fused-ring building blocks with the non-fused units.

In very recent years, great efforts have been devoted to designing the non-fused NFAs ${ }^{32-38}$, and the highest PCE of the OPV cells based on the completely non-fused NFAs has reached $12.76 \%{ }^{39}$, which is still obviously lower than the state-of-the-art results for the fused-ring NFAs ${ }^{40,41}$. Referring to these reported works, it can be concluded that the main challenge lies in controlling the molecular geometry and packing mode of the nonfused NFAs, but the method to overcome the challenge has not been well established. From the point of view of the molecular design of non-fused acceptors, the planarity and rigidity are crucial to the aggregation and charge transfer of the acceptors. On the one hand, the more planar configuration of the molecule ensures the effective $\pi-\pi$ stacking between the acceptors. On the other hand, the enhanced rigidity of the molecule can reduce the rotation of the $\mathrm{C}-\mathrm{C}$ single bond and improve the conformational stability of the acceptors. Unlike the planar and rigid fused-rings, the non-fused units consisting of two or more small aromatic rings can be easily twisted. Therefore, in terms of molecular geometry control, the functional groups that can induce the formation of noncovalent intramolecular interactions (such as $\mathrm{O} \cdots \mathrm{S}, \mathrm{F} \cdots \mathrm{S}$, and $\mathrm{N}$...S.) were commonly used to construct the planar non-fused units with higher conformation stability, which effectively enhances the photovoltaic properties of the completely non-fused NFAs such as BTIC-EH ${ }^{35}, \mathrm{Ph}^{-I^{32}}{ }^{2} \mathrm{PTIC}^{33}, \mathrm{o}-4 \mathrm{TBC}-$
$2 \mathrm{~F}^{34}$, etc. However, for realizing the aforementioned molecular packing mode, the functional groups with strong noncovalent intramolecular interaction cannot provide the high steric hindrance as the alkyls in the fused-ring units. Therefore, encouraged by the scientific mysteries of molecular design and the urgency of reducing the synthetic cost, it is of great necessity to develop a new method to ensure a quite planar and rigid non-fused conjugated structure and also simulate the steric hindrance of the alkyls in the fused-ring building blocks, based on which the nonfused NFAs with high photovoltaic performance will be expected.

In this contribution, we put forward a molecular design strategy by which a new bithiophene-based unit, 3,3'-bis(2,4,6-triisopropylphenyl)-2,2'-bithiophene (TT-P $i$ ), featuring good planarity, high conformational stability, and large steric hindrance, was obtained for forming the above-mentioned favourable molecular packing mode. Based on TT-P $i$, a new non-fused NFA $\left(2,2^{\prime}-\left(\left(\left(3,3^{\prime \prime \prime}\right.\right.\right.\right.$-bis(2-ethylhexyl)- $3^{\prime \prime}, 4^{\prime}$-bis(2,4,6-triisopropylphenyl)-[ $\alpha$-quaterthiophene $]-5,5^{\prime \prime}$ '-diyl)bis(methaneylylidene))bis(5,6-difluoro-3-oxo-2,3-dihydro- $1 \mathrm{H}$ indene-2,1-diylidene))dimalononitrile, A4T-16) was designed and synthesized, which has an optical bandgap $\left(E_{\mathrm{g}}{ }^{\mathrm{opt}}\right)$ of $1.45 \mathrm{eV}$ and a lowest-unoccupied-molecular-orbital (LUMO) level of $-3.96 \mathrm{eV}$. The single-crystal X-ray diffraction (XRD) results reveal that $\mathrm{A} 4 \mathrm{~T}$ 16 can form a 3D-interpenetrated network through the $\pi-\pi$ interaction of its end-groups, which facilitates electron transport. The spin-coated film of A4T-16 exhibits a relatively high electroluminescence (EL) efficiency, implying a low non-radiative energy loss in nature. Due to these favourable properties, the A4T-16-based cells yield an outstanding PCE of $15.2 \%$, which is not only the highest value among the OPV cells based on the non-fused electron acceptors but also at the same level as that of the fused-ring NFAs. What is more, in order to clearly demonstrate the critical role of TT$\mathrm{P} i$ in realizing the high-performance non-fused NFA, we designed and synthesized the analogues of TT-P $i$ and A4T-16 and carried out a series of comparative studies, which provide important information for the molecular design of other non-fused NFAs.

\section{Results and discussion}

Molecular design. In the established molecular design strategies, reducing the steric hindrance or enhancing the noncovalent intramolecular interaction between two non-fused aromatic rings has been widely used to obtain the planar conjugated structures. Here, we tried to realize the planar structure from the opposite direction, i.e., to obtain a planar structure with high conformational stability by introducing the functional groups with very large steric hindrance. We introduced two bulky functional groups, 2,4,6-tri-isopropylphenyl, onto the 3 - and 3 -position of a bithiophene unit to get TT-P $i$, and then analyzed the optimal geometry of TT-P $i$ by quantum chemistry calculation. As shown in Fig. 1a, the dihedral angel between the two thiophenes is $0^{\circ}$, implying the two thiophenes should be in one plane.

In order to prove the necessity of 2,4,6-tri-isopropylphenyl for achieving the high planarity, we calculated the optimal geometries of three representative bithiophene derivatives including bithiophene (TT), 3,3'-dimethoxy-bithiophene (TT-O) and 3,3'-difluoro-bithiophene (TT-F), and the other three analogues of TT-P $i$, including 3,3'-diphenyl-bithiophene (TT-P), 3,3'-bis(2,4,6-trimethoxylphenyl)-bithiophene (TT-Pom) and 3,3'-bis(2,4,6-trimethylphenyl)bithiophene (TT-Pm). As shown in Fig. la for their optimal geometries, TT shows a dihedral angel of $23.7^{\circ}$, after being substituted by fluorine or methoxy, the planar conjugated structure can be formed due to the noncovalent intramolecular interactions. For TT-P and TT-Pom, the dihedral angels between the adjacent thiophenes are $39.1^{\circ}$ and $36.3^{\circ}$, respectively, and these values between phenyl and thiophene are $60.2^{\circ}$ and $56.9^{\circ}$, respectively. However, for TT-Pm, due to the steric hindrance caused by the 


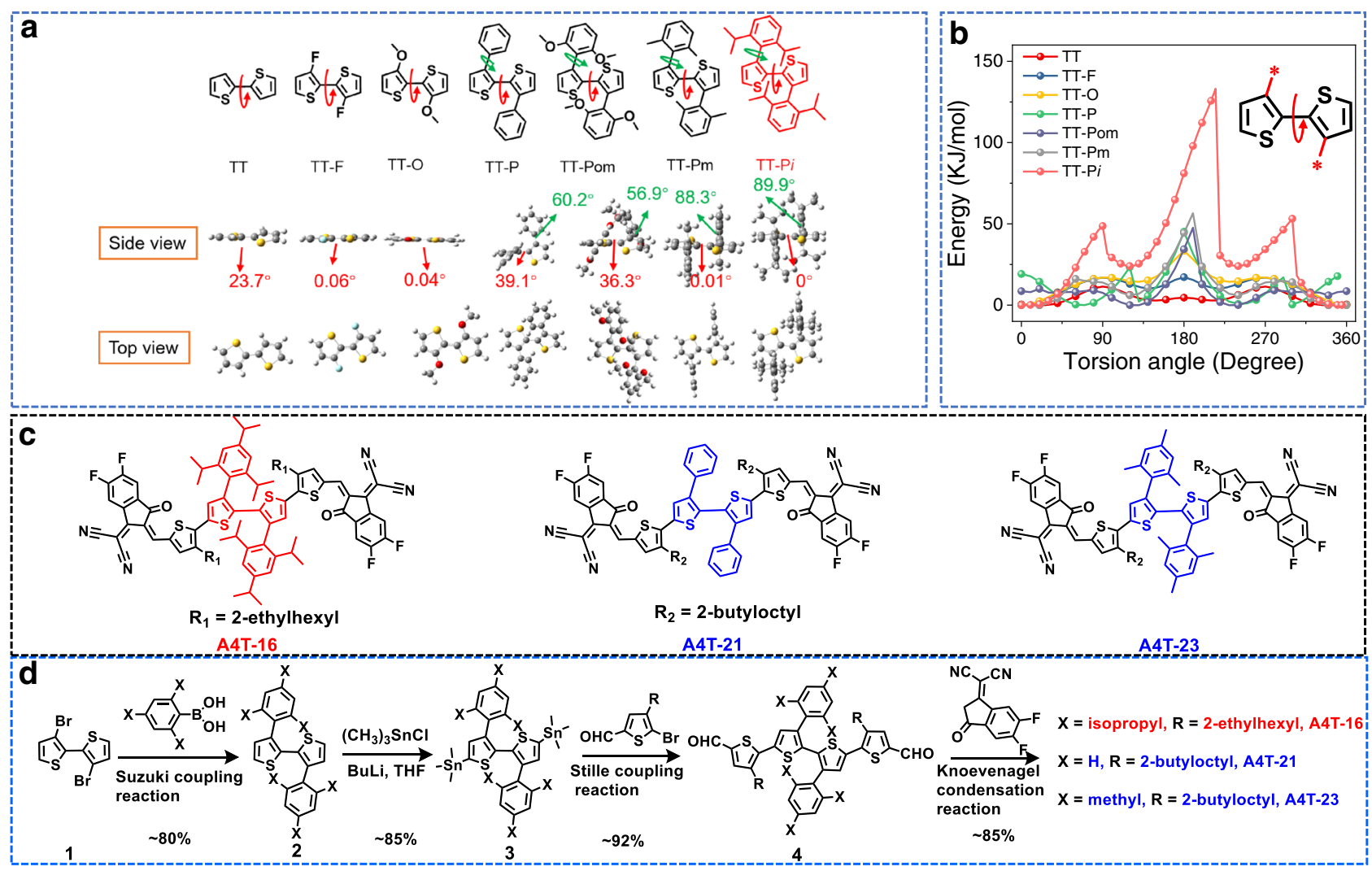

Fig. 1 Materials design and density functional theory (DFT) calculation results. a Chemical structures and DFT-calculated torsion angles of the optimal geometries for several bithiophene units at the B3LYP/6-31 G (d, p) level. b Torsional energy profiles between two thiophenes for several bithiophene units. c Chemical structures of A4T-16, A4T-21, and A4T-23. d Synthetic route of A4T-16, A4T-21, and A4T-23.

methyl groups, the dihedral angel between phenyl and thiophene enlarges to $88.3^{\circ}$, and the torsion angle between the two thiophenes becomes smaller, i.e., $0.01^{\circ}$. In TT-Pm, the phenyls are nearly perpendicular to bithiophene.

In addition, we also conducted the relaxed potential surface energy scans to evaluate the conformational stabilities of the rotatable C-C bonds between the thiophenes in the seven units. As depicted in Fig. 1b, for TT, the energy difference between the stable and meta-stable states $\left(\Delta E_{\mathrm{s}-\mathrm{ms}}\right)$ is about $3.0 \mathrm{~kJ} \mathrm{~mol}^{-1}$ and the rotation barrier from stable state to meta-stable state $\left(E_{s \rightarrow \mathrm{ms}}\right)$ is about $11 \mathrm{~kJ} \mathrm{~mol}^{-1}$. For TT-O and TT-F, due to the formation of the noncovalent intramolecular interaction, both $\Delta E_{\mathrm{s}-\mathrm{ms}}$ and $E_{s \rightarrow \mathrm{ms}}$ become larger, implying these two units are more prone to maintain the optimal geometries and also have higher conformational stabilities than TT. The calculation results also reveal that to enhance the steric hindrance caused by the substituents on 2and 4-position of the phenyl is the key to get the good planarity and high conformation stability in TT-P $i$. For TT-P $i, \Delta E_{s-m s}$ and $E_{s \rightarrow \mathrm{ms}}$ reach 24 and $48 \mathrm{~kJ} \mathrm{~mol}^{-1}$, respectively, which are much larger than those of the other six TT derivatives, implying the planar bithiophene structure in TT-P $i$ has very high conformational stability. Moreover, as shown in Fig. 1a for Van der Waals surface of TT-P $i$ with the optimal geometry, the two phenyls are perpendicular to the bithiophene, and almost the whole conjugated van der Waals surface of bithiophene is shielded by the isopropyl groups due to the large steric hindrance effect of the isopropyl groups (Supplementary Fig. 2); therefore, when TT-P $i$ is used as the core unit in an A-D-A-structured NFA, it can induce the formation of intermolecular $\pi-\pi$ stacking between the end groups.

Then, we designed a non-fused NFA, namely A4T-16. The molecular structure and synthetic route are shown in Fig. 1c, d.
Starting with 3,3'-dibromo-2,2'-bithiophene (compound 1), TT-P $i$ is prepared by Suzuki coupling reaction with a high yield of $80 \%$ according to the previous literature ${ }^{42-44}$. Subsequently, a typical procedure for introducing trimethyltin groups is adopted to prepare 5,5'- bis(trimethylstannyl)-3,3'-bis(2,4,6-triisopropylphenyl)[ $\alpha$-bithiophene] (compound 3) with a yield of $85 \%$. Then, 3,3'1'-bis(2ethylhexyl)- $3^{\prime \prime}, 4^{\prime}$-bis(2,4,6-triisopropylphenyl)-[ $\alpha$-quaterthiophene]5,5'I'-dicarbaldehyde (compound 4) can be synthesized by Stille coupling reaction between compound 3 and 5-bromo-4-(2-ethylhexyl) thiophene-2-carbaldehyde with a high yield of $92 \%$. Finally, A4T-16 is synthesized by Knoevenagel condensation between compound 4 and 2-(5,6-difluoro-3-oxo-2,3-dihydro-1H-inden-1ylidene)malononitrile with a yield of $85 \%$. A4T-16 can be easily dissolved in the common solvents such as chlorobenzene, chloroform, dichloromethane, toluene, o-xylene etc. What is more, in order to prove the advantages of the chemical structure of TT-P $i$, we also designed two analogue materials, A4T-21 and A4T-23, which are synthesized by the same synthetic route as that for A4T-16. The solubilities of A4T-16, A4T-21, and A4T-23 in o-xylene are 14, 90, and $18 \mathrm{mg} / \mathrm{mL}$, respectively. The detailed synthetic methods are provided in the "Experimental" section.

Quantum chemistry calculation. To demonstrate the optimal geometry of the three NFAs, the theoretical calculations were performed via DFT calculations at the B3LYP/6-31 G (d, p) level. The ethyl-hexyl/butyl-octyl side chains were simplified to isobutyl groups. For A4T-21, the backbone is highly twisted, two end groups are in different planes, while for A4T-16 and A4T-23, although the central cores are quite planar, torsions between the core (TT-P $i$ ) and the alkylthiophene are observed with torsional angles of $28.4^{\circ}$ and $30.1^{\circ}$, respectively. Besides, for A4T-16 and 


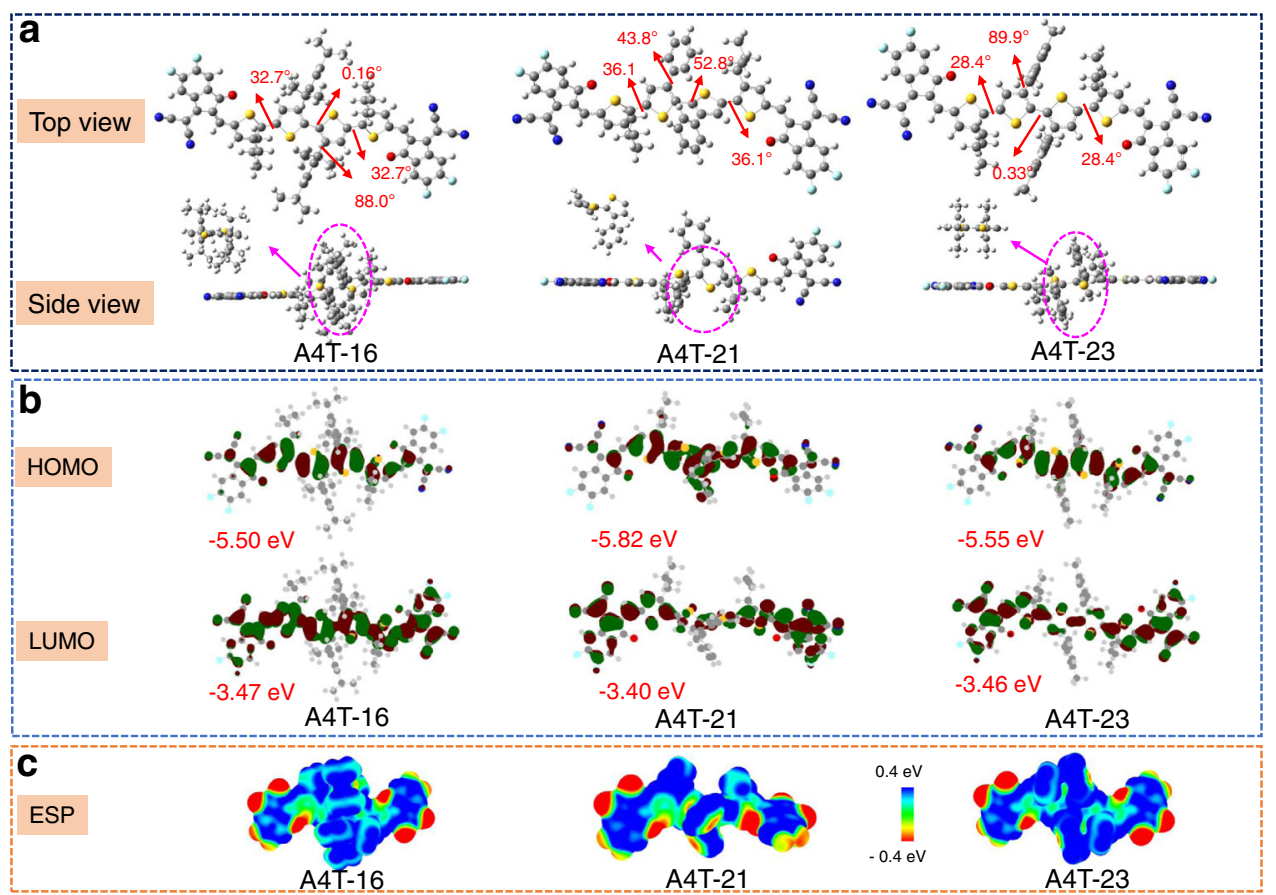

Fig. 2 DFT calculation results of A4T-16, A4T-21, and A4T-23. a The optimal geometries. b DFT-calculated frontier molecular orbitals. c ESP distributions for the simplified structures of A4T-16, A4T-21, A4T-23 at the B3LYP/6-31 G(d,p) level.

A4T-23, the phenyl groups are nearly perpendicular to the bithiophene with dihedral angles of $88.0^{\circ}$ and $89.9^{\circ}$, respectively (Fig. 2a). From the calculation results, we can roughly infer that the highly twisted conjugated backbone of A4T-21 may adversely affect the molecular packing. The calculated distribution of the frontier molecular orbitals of the three acceptors is shown in Fig. 2b. The highest occupied molecular orbital (HOMO) and LUMO surface of them are well delocalized over the whole conjugated backbones, the calculated values are $-5.50 /-3.47$, $-5.82 /-3.40,-5.55 /-3.46 \mathrm{eV}$ for A4T-16, A4T-21, and A4T-23, respectively. Moreover, their electrostatic potential (ESP) distributions are also displayed in Fig. 2c, the high ESP values suggest their excellent electron-accepting capacity. Overall, theoretical investigations manifest that A4T-16 and A4T-23 are more likely to adapt planer backbone than that of A4T-21 as the preferential conformation, which would be beneficial to ICT and intermolecular charge transport.

Single crystal structure. Single crystallographic data provide solid information for the determination of the molecular geometries and intermolecular stacking modes of organic compounds and thus have been successfully used to get insights into the correlations between aggregation structures and charge transport properties of the fused-ring-based $\mathrm{NFAs}^{30,45}$. After many attempts, we got the single crystal of A4T-16 via vapour diffusion method. As shown in Fig. 3a, for the single molecular configuration of A4T-16, the dihedral angle between the phenyl and thiophene is $89.9^{\circ}$ and the dihedral angle between the two thiophenes in the core is $0.13^{\circ}$, which agrees well with the calculation results for TT-Pi; the alkylthiophene is twisted relative to TT-P $i$, with a dihedral angle of $30.1^{\circ}$, and the dihedral angle between the alkylthiophene and the end group is $1.51^{\circ}$. In a single crystal, A4T-16 shows two orthogonal orientations (coloured with green and orange in Fig. 3b-e). As shown in Fig. 3b, in the in-plane horizontal direction, two adjacent A4T-16 molecules stack with each other through the end-capping groups and form a straight linear sub-structure in the crystal; by the same mode, such a sub-structure also forms in the out-plane horizontal direction. So, in these two directions, the electrons can be easily transported. The linear sub-structures in the two directions interact with each other through the end-capping groups (Fig. 3c) and form an interlocked network structure (Fig. 3d, e). In this network structure, the $\pi-\pi$ distances between the end-capping groups are $3.35 \AA$, which is very compact and even smaller than those in the typical Y6-type and ITIC-type molecules ${ }^{29-31}$. Therefore, the crystalline structure of A4T-16 can be seen as an ideal 3D interpenetrating network for electron transport, and TTPi plays a critical role in forming such a network.

Photophysical and electrochemical properties. The ultravioletvisible (UV-vis) absorption spectra of the three NFAs in solid films and solution are shown in Fig. 4a and Supplementary Fig. 3, respectively. In dilute chloroform solution, the absorption spectrum of A4T-16 is almost the same as that of A4T-23, implying $\pi$ electron delocalization and molecular geometry of A4T-16 are very similar to those of A4T-23, which agree with the quantum calculation results. The molar extinction coefficients of A4T-16, A4T-21, and A4T-23 are $8.9 \times 10^{4}, 7.2 \times 10^{4}$, and $1.2 \times 10^{5} \mathrm{M}^{-1}$ $\mathrm{cm}^{-1}$ at $686,601,686 \mathrm{~nm}$, respectively (Supplementary Fig. $3 \mathrm{c}$ ). In the solid neat films, the absorption spectra of the three materials are red-shifted. For A4T-16, the film shows an absorption peak at $756 \mathrm{~nm}$ and an absorption edge at about $855 \mathrm{~nm}$, corresponding to an optical bandgap ( $\left.E_{\mathrm{g}}{ }^{\text {opt}}\right)$ of $1.45 \mathrm{eV}$. $E_{\mathrm{g}}{ }^{\text {opt }}$ value of A4T- 16 is slightly smaller than that of IT- $4 \mathrm{~F}^{46}$ but obviously larger than $\mathrm{Y}^{8}$, these two are broadly used as fusedring-based NFAs. The extinction coefficients of A4T-16, A4T-21, and A4T-23 films are $7.70 \times 10^{4}, 6.76 \times 10^{4}$, and $8.23 \times 10^{4} \mathrm{~cm}^{-1}$, respectively (Fig. 4a). Obviously, the absorption spectra of A4T21 show a large hypsochromic shift than that of A4T-16 and A4T-23, which mainly resulted from the twisted backbone of A4T-21 that suppressed the ICT effect ${ }^{47-49}$. The detailed absorption spectral parameters of A4T-16, A4T-21, and A4T-23 are provided as Supplementary Information (SI, Supplementary Table 1). Electrochemical cyclic voltammetry (CV) measurements 


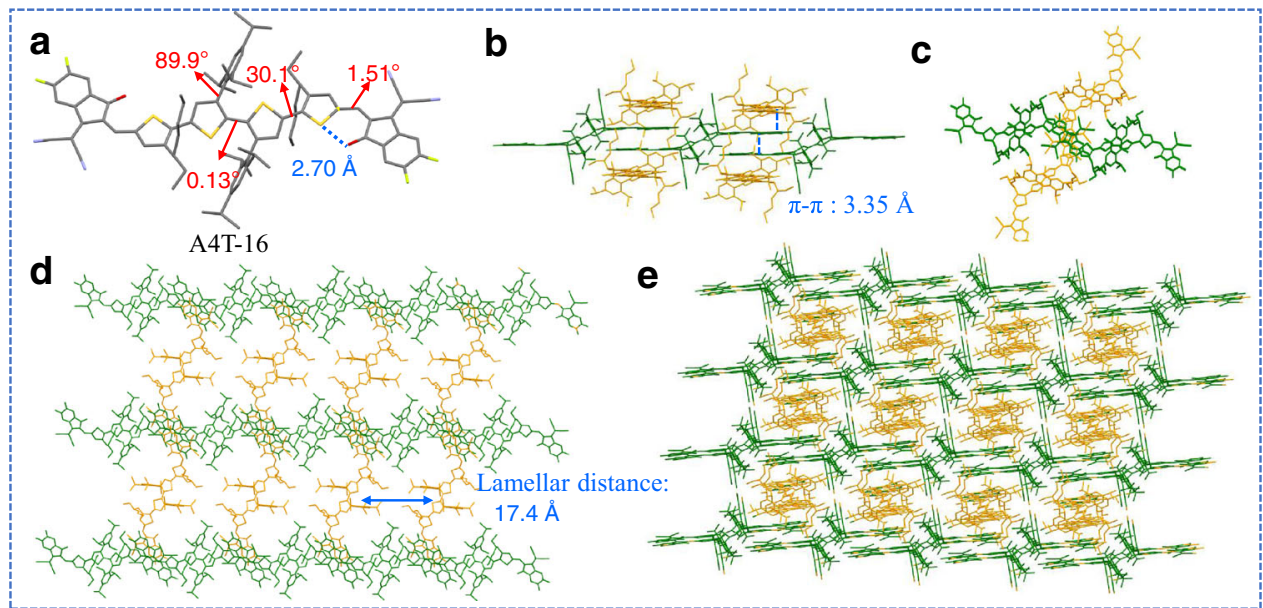

Fig. 3 Single-crystal structure of A4T-16. a Single-crystal structure of A4T-16. b Molecules packing viewed from the $x-z$ plane. c Molecules packing viewed from the $x-y$ plane. $\mathbf{d}$ The top view of the network structure of A4T-16. e 3D interpenetrating network structure of A4T-16.
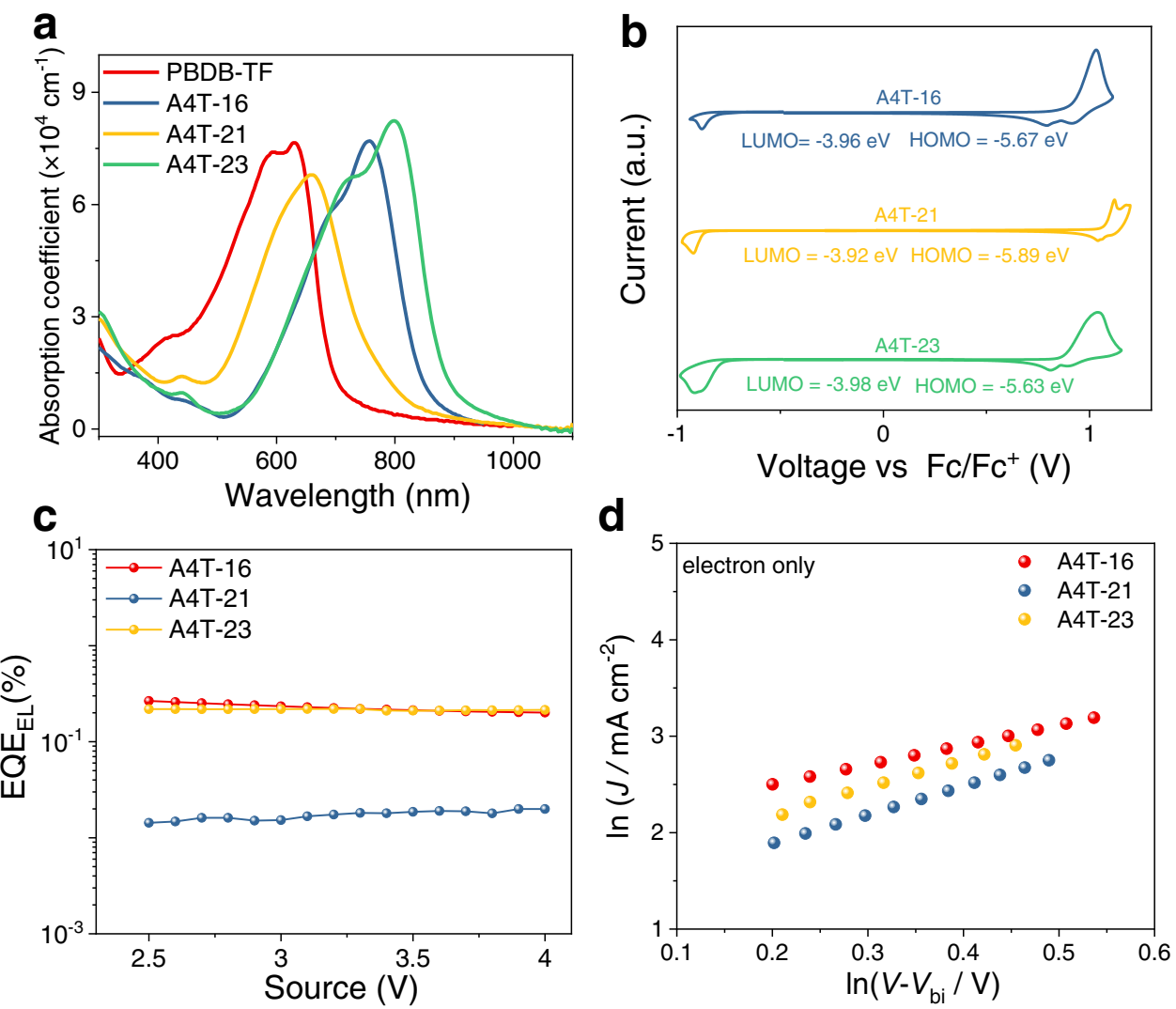

Fig. 4 Photophysical and electrochemical properties of NFAs. a UV-vis absorption spectra of PBDB-TF, A4T-16, A4T-21, and A4T-23 in film states. b Cyclic voltammograms of A4T-16, A4T-21, and A4T-23. c EQE $E_{E L}$ curves of A4T-16, A4T-21 and A4T-23. d The electron mobility plots of the three acceptors.

were performed to determine the LUMO levels of the acceptors, and as demonstrated in Fig. 4b, the LUMO levels of A4T-16, $\mathrm{A} 4 \mathrm{~T}-21$, and $\mathrm{A} 4 \mathrm{~T}-23$ are $-3.96-3.92$, and $-3.98 \mathrm{eV}$, respectively, which are slightly higher than that of IT-4F and Y6 (see Supplementary Fig. 4 for the CV curves). Overall, these three materials should be good acceptors in terms of the absorption spectra and the LUMO levels. From the point of view of molecular energy level and absorption spectrum matching, PBDB-TF, a broadly used donor material, should be a good candidate to work with the three non-fused NFAs in OPV cells.
In recent studies ${ }^{50,51}$, external quantum efficiencies of EL $\left(\mathrm{EQE}_{\mathrm{EL}}\right)$ were employed to evaluate the non-radiative energy loss $\left(\Delta E_{\text {non-rad }}\right)$ of photovoltaic cells, because the cells with higher $\mathrm{EQE}_{\mathrm{EL}}$ and thus relatively lower $\Delta E_{\text {non-rad }}$ have more potential in realizing higher PCEs. It has also been recognized that the $\mathrm{EQE}_{\mathrm{EL}}$ of an OPV cell can be influenced by the interaction between the donor and acceptor materials, but its maximum $\mathrm{EQE}_{\mathrm{EL}}$ value depends more on the component with the relatively narrower bandgap. Therefore, $\mathrm{EQE}_{\mathrm{EL}}$ of the three non-fused NFAs were measured and then compared with those of the two 


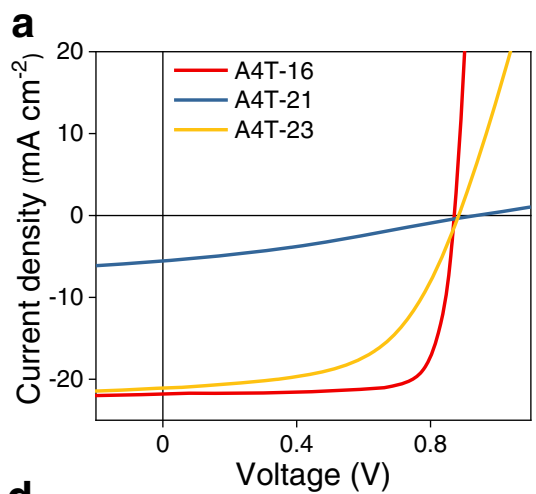

b
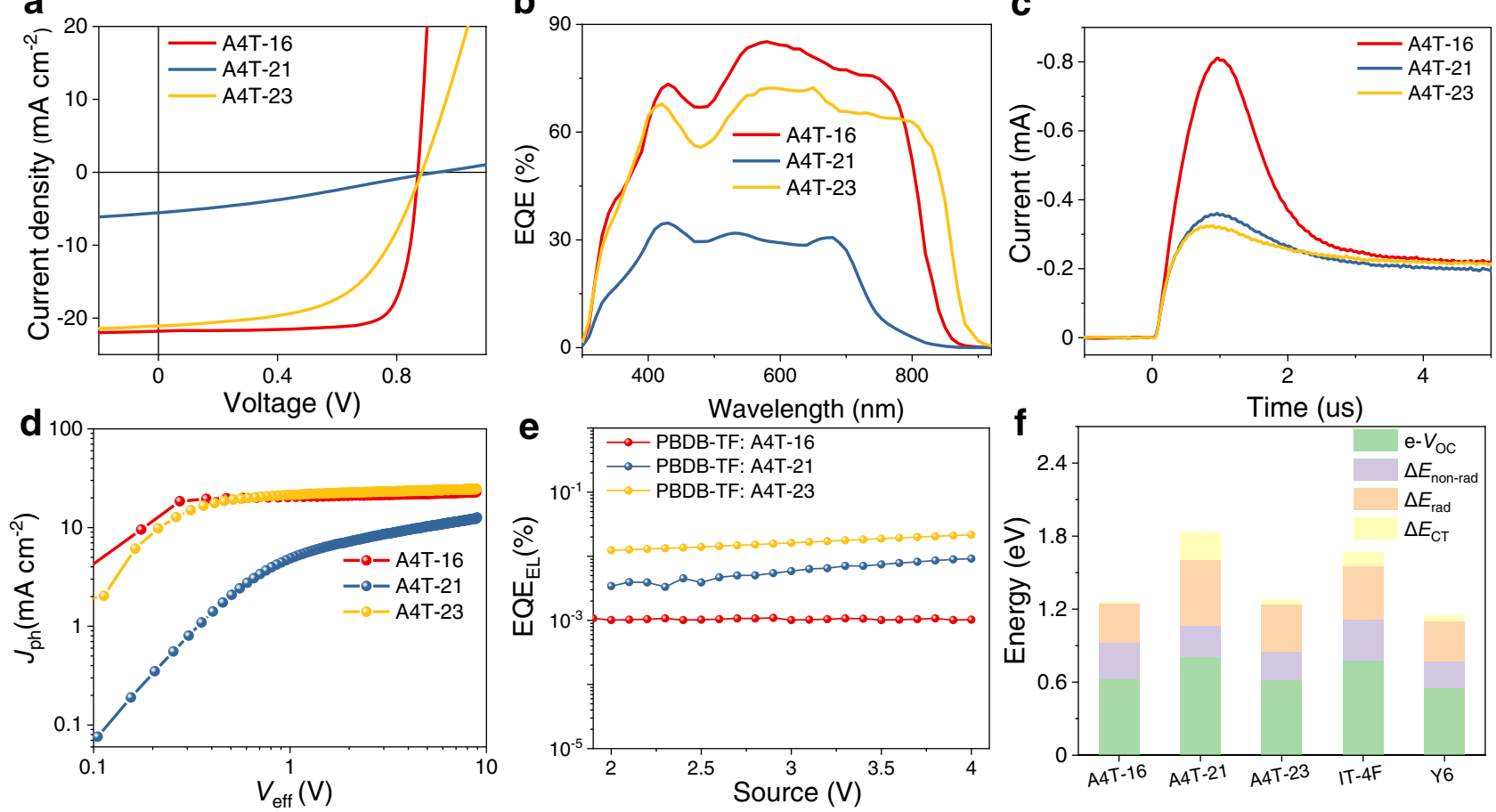

Fig. 5 Photovoltaic properties, and recombination mechanism of the OPV cells. a $J-V$ curves, b EQE curves, c (Photo-CELIV) measurements for the mobilities of the fast carrier, $\mathbf{d} \mathrm{J}_{\mathrm{ph}}-\mathrm{V}_{\text {eff }}$ curves, $\mathbf{e} \mathrm{EQE}_{\mathrm{EL}}$ curves of A4T-16-, A4T-21-, and A4T-23-based cells. f Energy losses of A4T-16-, A4T-21-, A4T-23-, IT-4F-, Y6-based cells.

representatives fused-ring-based NFAs, IT-4F, and Y6. As demonstrated in Fig. 4c and Supplementary Fig. 5a, A4T-16 and A4T-23 show a high $\mathrm{EQE}_{\mathrm{EL}}$ of around $2.5 \times 10^{-3}$ (Fig. $4 \mathrm{c}$ ), and this value is very similar to that of $\mathrm{Y} 6\left(2.3 \times 10^{-3}\right)$ (Supplementary Fig. 5a). By contrast, $\mathrm{EQE}_{\mathrm{EL}} \mathrm{S}$ of $\mathrm{A} 4 \mathrm{~T}-21$ and IT-4F are $1.5 \times 10^{-4}$ and $2.1 \times 10^{-4}$, respectively, which are about one order of magnitude lower than that of A4T-16. The high $\mathrm{EQE}_{\mathrm{EL}}$ of $\mathrm{A} 4 \mathrm{~T}-16$ implies that it has much potential in realizing OPV cells with low $E_{\text {loss }}$ values. In addition, we measured the electron mobilities $\left(\mu_{\mathrm{e}}\right)$ of these acceptors in parallel by spacecharge-limited-current (SCLC) measurement ${ }^{52}$ (see Fig. $4 \mathrm{~d}$, Supplementary Fig. 5b and Supplementary Table 2) and found that $\mu_{\mathrm{e}} \mathrm{s}$ of A4T-16 and A4T-23 are $5.5 \times 10^{-5}$ and $2.7 \times 10^{-5} \mathrm{~cm}^{2}$ $\mathrm{V}^{-1} \mathrm{~S}^{-1}$, which are quite similar to that of IT-4F $\left(5.9 \times 10^{-5} \mathrm{~cm}^{2}\right.$ $\left.\mathrm{V}^{-1} \mathrm{~S}^{-1}\right)$ and Y6 $\left(5.4 \times 10^{-5} \mathrm{~cm}^{2} \mathrm{~V}^{-1} \mathrm{~S}^{-1}\right)$, while $\mu_{\mathrm{e}}$ of A4T-21 is about one to two times lower than them due to the twisted backbone and the poor molecular $\pi-\pi$ stacking. Therefore, in terms of $\mathrm{EQE}_{\mathrm{EL}}$ and electron mobility, A4T-16 and A4T-23 have more potential to be good NFAs.

Photovoltaic properties, and recombination mechanism. To evaluate the photovoltaic performance of these non-fused acceptors, we fabricated the OPV cells with a device architecture of ITO/PEDOT: PSS/active layer $/ \mathrm{PF}_{3} \mathrm{~N}-\mathrm{Br} / \mathrm{Ag}$. In the active layers, PBDB-TF was blended with A4T-16, A4T-21, and A4T-23, respectively. In parallel, the PBDB-TF:IT-4F- and PBDBTF:Y6-based cells were fabricated by following the reported device fabrication procedures and used as the control devices 8,53 . The device fabrication conditions of the cells based on the three new NFAs are optimized by the commonly used processes. Here, we put the photovoltaic results of the cells fabricated via the optimal conditions together for detailed discussion.

For the cells based on the three new non-fused NFAs, the current density-voltage $(J-V)$ curves and the key parameters including PCE, open-circuit voltage $\left(V_{\mathrm{OC}}\right)$, short circuit current density $\left(J_{\mathrm{SC}}\right)$ and fill factor $(\mathrm{FF})$ are shown in Fig. $5 \mathrm{a}$ and Table 1. For the control samples, the IT-4F-and Y6-based cells exhibit PCEs of $13.7 \%\left(V_{\mathrm{OC}}=0.852 \mathrm{~V} ; J_{\mathrm{SC}}=21.3 \mathrm{~mA} \mathrm{~cm}^{-2} ; \mathrm{FF}=0.757\right)$ and $15.8 \% \quad\left(V_{\mathrm{OC}}=0.841 \mathrm{~V} ; J_{\mathrm{SC}}=25.5 \mathrm{~mA} \mathrm{~cm}^{-2} ; \quad \mathrm{FF}=0.737\right)$ (Supplementary Table 3 ), and these photovoltaic parameters are in accordance with the reported results in literatures ${ }^{8,46}$. For the three non-fused NFA-based devices, the A4T-21-based cell demonstrates the highest $V_{\mathrm{OC}}$ of $0.936 \mathrm{~V}$ but the lowest PCE of $1.57 \%$ due to the inferior $J_{\mathrm{SC}}\left(5.55 \mathrm{~mA} \mathrm{~cm}^{-2}\right)$ and FF $(0.303)$. The A4T-23-based cell delivers a PCE of $10.4 \%$ accompanying with a $V_{\mathrm{OC}}$ of $0.870 \mathrm{~V}$, a $J_{\mathrm{SC}}$ of $21.0 \mathrm{~mA} \mathrm{~cm}^{-2}$, and an FF of 0.568 , and such a PCE is at the same level as the state-of-the-art cells based on the completely non-fused NFAs ${ }^{34,36}$. Encouragingly, an impressive PCE of $15.2 \%\left(V_{\mathrm{OC}}=0.876 \mathrm{~V}, J_{\mathrm{SC}}=21.8 \mathrm{~mA} \mathrm{~cm}^{-2}\right.$, $\mathrm{FF}=0.798)$ is recorded for the A4T-16-based cell, which is certified as $14.8 \%$ by the National Institute of Metrology, China. (Supplementary Fig. 7). The result is not only the highest one for the non-fused NFAs but also higher than the IT-4F-based control sample. Notably, in comparison with the Y6-based control sample, the $J_{\mathrm{SC}}$ of the A4T-16-based cell is lower, because A4T16 has a larger $E_{\mathrm{g}}{ }^{\text {opt }}$ than that of $\mathrm{Y} 6$, which limits the sunlight absorption capability of the A4T-16-based cell. Considering the simpler structure and the relatively high performance of these acceptors, we calculated the figure-of-merits (FoM) values of these NFAs and found that A4T-16 processes higher FoM value among these high-performance NFAs (Supplementary Table 7).

The external quantum efficiency (EQE) spectra of the cells are shown in Fig. 5b and Supplementary Fig. 6b. The integrated $J_{\mathrm{SC}}$ values are $21.1,6.91,20.2,20.2$ and $25.3 \mathrm{~mA} \mathrm{~cm}^{-2}$ for the A4T16-, A4T-21-, A4T-23-, IT-4F-, Y6-based devices, respectively, which are in accordance with those from $J-V$ measurements. For the A4T-21-based cell, the relatively narrower response range should be ascribed to the light absorption capability of A4T-21, and the low EQE values indicate severe charge recombination in 
the cell. What is more, the integral current density of the A4T-21based device is much higher than that obtained in the $J-V$ curve; such a phenomenon indicates the hole and electron channels are not well-formed in the cell, so the severe charge recombination can be reduced to a certain degree under the weaker light intensity condition like in EQE measurement. In comparison with the A4T-16-based cell, the A4T-23-based cell shows a broader response range, which is in accord with the observations in their absorption spectra, i.e., the absorption peak of A4T-23 locates at the longer wavelength position than that of A4T-16. As the FF and EQE of the A4T-16-based cell are quite similar to the two control samples with disregarding light response range, we can conclude that charge generation and transport in the A4T-16based cell should be efficient.

To understand the reason for the difference of the photovoltaic parameters in the three devices based on the non-fused NFAs, we analyzed their carrier transport and recombination properties. The photocurrent density $\left(J_{\mathrm{ph}}\right)$ versus effective voltage $\left(V_{\text {eff }}\right)$ curves were used to evaluate the charge dissociation probabilities $\left(P_{\text {diss }}\right)$ in the cells ${ }^{54}$. The saturated $J_{\mathrm{ph}}$ of the A4T-16- and A4T23-based cells are 22.9 and $24.6 \mathrm{~mA} \mathrm{~cm}^{-2}$ with $P_{\text {diss }}$ of 95 and $85 \%$, respectively (Fig. $5 \mathrm{~d}$ ); the $J_{\mathrm{ph}}$ values are quite high for their light absorption ranges and signify that the light-induced charge generation in the two cells are equally efficient while the charge recombination in the A4T-16-based cell is obviously lower than that in the A4T-23-based cell. For the A4T-21-based cell, the $J_{\mathrm{ph}}$ is not saturated until the $V_{\text {eff }}$ reaches $10 \mathrm{~V}$ and the $P_{\text {diss }}$ value is quite low $(43.3 \%)$, which indicates inefficient charge generation and strong charge recombination. We then measured the dependence of $J_{\mathrm{SC}}$ and $V_{\mathrm{OC}}$ on light intensity $\left(P_{\text {light }}\right)$ to investigate the charge recombination in these cells. As plotted in Supplementary Fig. 8, the recombination parameter $s$ in the formula $J_{\mathrm{SC}}$ $\propto P_{\text {light }}{ }^{\mathrm{s}}$ is 0.996 for the A4T-16-based cell, implying negligible bimolecular recombination; for the A4T-23-based cell, the bimolecular recombination is slightly stronger with as value of 0.950, while for the A4T-21-based cell, a much smaller s of 0.835 indicates severe bimolecular recombination, which explains the inferior $J_{\mathrm{SC}}$ and PCE of the cell. In addition, the slopes $\left(\mathrm{s}^{\prime}\right)$ obtained from the equation of $V_{\mathrm{OC}} \propto s^{\prime} k_{\mathrm{B}} T / q \ln \left(P_{\text {light }}\right)$ can be used as the indicator for estimating trap-assisted recombination ${ }^{55}$. As the three cells show similar slopes between 1.0 and 1.1, the trapassisted recombination in these cells is negligible when the light intensity varies from 1 to $100 \mathrm{~mW} \mathrm{~cm}^{-2}$ (Supplementary Fig. 8).

To assess the charge transport properties in the three cells, we performed the photo-induced charge-carrier extraction in a linearly increasing voltage (photo-CELIV) measurements. As displayed in Fig. $5 c$, the mobilities of the faster carriers $\left(\mu_{\text {celiv }}\right)$ for the A4T-16- and A4T-23-based cells are $2.4 \times 10^{-4}$ and $3.0 \times 10^{-4} \mathrm{~cm}^{2} \mathrm{~V}^{-1} \mathrm{~S}^{-1}$, respectively, which are at the same level as those of the highly efficient cells in previous works ${ }^{56,57}$, while $\mu_{\text {celiv }}$ of the A4T-21-based cell $\left(1.9 \times 10^{-4} \mathrm{~cm}^{2} \mathrm{~V}^{-1} \mathrm{~S}^{-1}\right)$ is lower than them (Table 1). Moreover, we also fabricated the holeand electron-only devices and measured the hole and electron mobilities $\left(\mu_{\mathrm{h}}\right.$ and $\left.\mu_{\mathrm{e}}\right)$ of the three non-fused NFA-based active layers by SCLC method. As shown in Supplementary Table 2 and Supplementary Fig. 9, for the A4T-21-based active layer, a much lower $\mu_{\mathrm{e}}\left(\mu_{\mathrm{e}}=1.2 \times 10^{-5} \mathrm{~cm}^{2} \mathrm{~V}^{-1} \mathrm{~S}^{-1}\right)$ than the other two active layers is obtained and also the hole and electron transport capabilities are asymmetric, which should be the main reasons for the severe bimolecular charge recombination effect and low FF of the cell; the A4T-16-based active layer displays a highly symmetric $\mu_{\mathrm{h}}$ and $\mu_{\mathrm{e}}$ of $3.0 \times 10^{-4}$ and $2.9 \times 10^{-4} \mathrm{~cm}^{2} \mathrm{~V}^{-1} \mathrm{~S}^{-1}$, respectively, which explains the low charge recombination and thus high EQE and FF of the corresponding cell. In addition, the miscibility of the blend film was investigated, and the Flory-Huggins interaction parameters $(\chi)$ of PBDB-TF:A4T-16, PBDB-TF:A4T-21 and 
PBDB-TF:A4T-23 were calculated to be $0.52,0.43$, and 0.41 , respectively (Supplementary Table 5 ). The smaller $\chi$ value indicates higher miscibility between PBDB-TF and A4T-23, which is not conducive to the formation of a purer domain. The results above can partially explain the inferior FF in PBDB-TF:A4T-23based device ${ }^{58,59}$.

The Urbach energies $\left(E_{\mathrm{U}} \mathrm{s}\right)$ can be utilized to evaluate the energetic disorder of the three blends by measuring the highly sensitive EQE (s-EQE) spectra ${ }^{60}$. The calculated $E_{\mathrm{U}} \mathrm{s}$ are $25.4,26.9$, $26.2 \mathrm{meV}$ for A4T-16-, A4T-21-, A4T-23-based devices (Supplementary Fig. 10a), smaller $E_{U}$ values means a lower disorder in the blend and higher potential for achieving lower energy losses. To get insights into the $V_{\mathrm{OC}}$ difference of the three non-fused NFA-based cells, we carried out EL and high-sensitive EQE measurements to investigate the causes of energy losses. Referring to the literatures $50,51,61$, the bandgaps $\left(E_{\mathrm{g}}\right)$ of the OPV cells based on the low bandgap NFAs can be determined by the cross point of the absorption and photoluminescence spectra of the NFAs. By this method, $E_{\mathrm{g}}$ of the A4T-16-, A4T-21- and A4T-23-based cells are calculated to be 1.51, 1.74, and $1.49 \mathrm{eV}$ (Supplementary Fig. 10b), respectively, so the overall voltage losses which can be calculated by the equation of $\mathrm{e} V_{\text {loss }}=E_{\mathrm{g}}-\mathrm{e} V_{\mathrm{OC}}$ for the three cells are 0.634 , 0.804 and $0.620 \mathrm{eV}$, respectively. Based on the $\mathrm{EQE}_{\mathrm{EL}}$ data shown in Fig. $5 \mathrm{e}$, the $\Delta E_{\text {non-rad }}$ are calculated to be $0.298,0.255$ and $0.227 \mathrm{eV}$ for the A4T-16-, A4T-21-, and A4T-23-based cells, respectively. As $\mathrm{e} V_{\text {loss }}-\Delta E_{\mathrm{CT}}$ can be separated into radiative and non-radiative energy loss ( $\Delta E_{\text {rad }}$ and $\left.\Delta E_{\text {non-rad }}\right)$, the $\Delta E_{\text {rad }}$ of the A4T-16-, A4T21-, and A4T-23-based cells are $0.326,0.329$ and $0.363 \mathrm{eV}$, respectively. The variation of $\Delta E_{\text {rad }}$ and $\Delta E_{\text {non-rad }}$ in the three blend films is a very interesting phenomenon, and the reason still needs to be revealed by combining in-depth theoretical calculation and optical physical characterization. In parallel, $\mathrm{e} V_{\text {loss }}-\Delta E_{\mathrm{CT}}$ $/ \Delta E_{\text {rad }} / \Delta E_{\text {non-rad }}$ of the IT-4F- and Y6-based cells are measured, which are $0.668 / 0.332 / 0.336 \mathrm{eV}$ and $0.499 / 0.273 / 0.226 \mathrm{eV}$, respectively (Supplementary Fig. 10c and Supplementary Table 6).

Overall, both the non-radiative and radiative energy loss of the A4T-16-based cell are lower than those of the IT-4F-based cell; in comparison with the Y6-based cell, the A4T-16-based cell shows larger radiative energy loss $(0.326 \mathrm{eV}$ versus $0.273 \mathrm{eV})$ and nonradiative energy loss $(0.298 \mathrm{eV}$ versus $0.226 \mathrm{eV})$. In consideration that the non-radiative energy loss of the neat A4T-16 film is at the same level as Y6 and the non-radiative energy losses of the D: A blend films can also be tuned by changing the interaction between the donor and acceptor materials ${ }^{62,63}$, there should be much room for reducing the non-radiative energy loss of the A4T-16based cells. For this purpose, PBDB-TF should be replaced by other polymer donor materials.

Morphological characteristics. Intermolecular packing structures of the three non-fused NFAs in the neat spin-coated films were analyzed by grazing incidence wide-angle X-ray scattering (GIWAXS). The 2D scattering patterns and the in-plane (IP) and out-of-plane (OOP) scattering profiles are illustrated in Fig. 6a, b, respectively. For the A4T-16 film, a weak (010) diffraction signal can be distinguished at $1.87 \AA^{-1}$ in both the OOP and IP directions, corresponding to very compact $\pi-\pi$ stacking distances of $3.35 \AA$; the periodical peaks at 0.36 and 0.72 are assigned to the (100) and (200) diffractions with a laminar packing distance of 17.4 $\AA$. These values are in agreement with the single-crystal structural results (Fig. 3). Notably, the observed annular diffraction pattern is similar to that of the fullerene acceptors, suggesting that the $3 \mathrm{D}$ network structure mentioned above will endow more electron channels for favourable charge transfer in this acceptor. For the A4T-21 film, a typical diffraction pattern for the multicrystal films can be observed, while (010) signal for $\pi-\pi$ stacking is absent in OOP and IP direction. For the A4T-23 film, the faceon orientation signified by a (010) diffraction peak at $1.82 \AA^{-1}$ at OOP direction and a (100) diffraction signal at $0.39 \AA^{-1}$ can be found, corresponding to a $\pi-\pi$ distance of $3.45 \AA$ and a laminar packing distance of $16.1 \AA$. The above results confirm that the $3 \mathrm{D}$ electron transport network observed in the A4T-16 single crystal can be formed in the A4T-16 film, and also indicate the nonfused cores had a great effect on the molecular crystallinities of these acceptors.

We also analyzed the morphological properties of the blend films of PBDB-TF:A4T-16, PBDB-TF:A4T-21, and PBDBTF:A4T-23. As shown in Fig. 6c, d for the PBDB-TF:A4T-16 blend film, the annular (100) diffraction pattern of A4T-16 still can be distinguished at the similar positions as that in the neat A4T-16 film, implying that the 3D-network of A4T-16 retained in this blend film in a certain degree. The PBDB-TF:A4T-21 films exhibited (100) lamellar peaks locating at $0.31 \AA^{-1}$ in both the IP and OOP directions, corresponding to a $d$-spacing of $20.3 \AA$, and (010) diffractions only in the OOP direction (Fig. 6d), indicating a face-on-dominated molecular orientation in the film. Unlike the above two films, PBDB-TF: A4T-23 prefers to form a mixed faceon and edge-on orientations with (010) peaks in OOP and IP directions. The (010) peaks of the PBDB-TF:A4T-16, PBDBTF:A4T-21, and PBDB-TF:A4T-23 films are at 1.87, 1.77, and $1.79 \AA^{-1}$, corresponding to the $\pi-\pi$ distances of $3.36,3.55$, and $3.51 \AA$, respectively. Obviously, the more compact $\pi-\pi$ distance can be formed in the PBDB-TF:A4T-16 film than the other two blends. The $\pi-\pi$ distance in PBDB-TF:A4T-23 blend film is larger than that of in A4T-23 neat film, implying the molecular orientation of A4T-23 is more significantly affected by PBDB-TF compared with A4T-16. The face-on orientation and $\pi-\pi$ packing distance of A4T-23 are not well retained in the A4T23-based blend film. In addition, the higher miscibility between PBDB-TF and A4T-23 aforementioned may also be responsible for the decreased FF in the OPV cell.

As depicted in Fig. 6a and Supplementary Fig. 12, by employing chloroform as the processing solvent for spin-coating, the neat A4T-16 film demonstrates high surface roughness $\left(R_{\mathrm{q}}\right)$ of $6.4 \mathrm{~nm}$ with large aggregations, while the A4T-21 and A4T-23 films show much smoother surfaces with the $R_{\mathrm{q}}$ of 2.8 and $0.5 \mathrm{~nm}$, respectively. The PBDB-TF:A4T-16-, PBDB-TF:A4T-21-, and PBDB-TF:A4T-23-based films demonstrate relatively smooth surfaces with the $R_{\mathrm{q}}$ of $2.5,4.1$, and $3.0 \mathrm{~nm}$ (Fig. $6 \mathrm{c}$ ), and the nanoscale phase separation can be formed in these blends (Supplementary Fig. 12b). Therefore, generally speaking, the three non-fused NFAs have no problem in realizing the favourable phased separation when blended with PBDB-TF. In transmission electron microscope (TEM) measurement, there was no significant difference in phase separation of the three non-fused blend films (Supplementary Fig. 13).

Universality and device stability of A4T-16. Here, we selected a few representative polymer donors including PBDB-T, J52-2F, PTO2 and PBDB-TCl (Supplementary Fig. 14) to blend with A4T-16 and then fabricated a series of OPV cells. The aforementioned device architecture and the same fabrication conditions for the PBDB-TF:A4T-16-based cell are adopted without any further optimization. The $J-V$ and $\mathrm{EQE}_{\mathrm{EL}}$ curves and the photovoltaic parameters are shown in Fig. $7 \mathrm{a}, \mathrm{b}$ and Table 2.

It should be also noted that, the device fabrication method that inherits from the PBDB-TF:A4T-16 cells may not be optimal for making the cells with the other polymers, so the photovoltaic results in this section can only be used to make a preliminary evaluation for the universality of A4T-16. Nevertheless, it is very clear that no matter which donor is used, the cells exhibit decent 

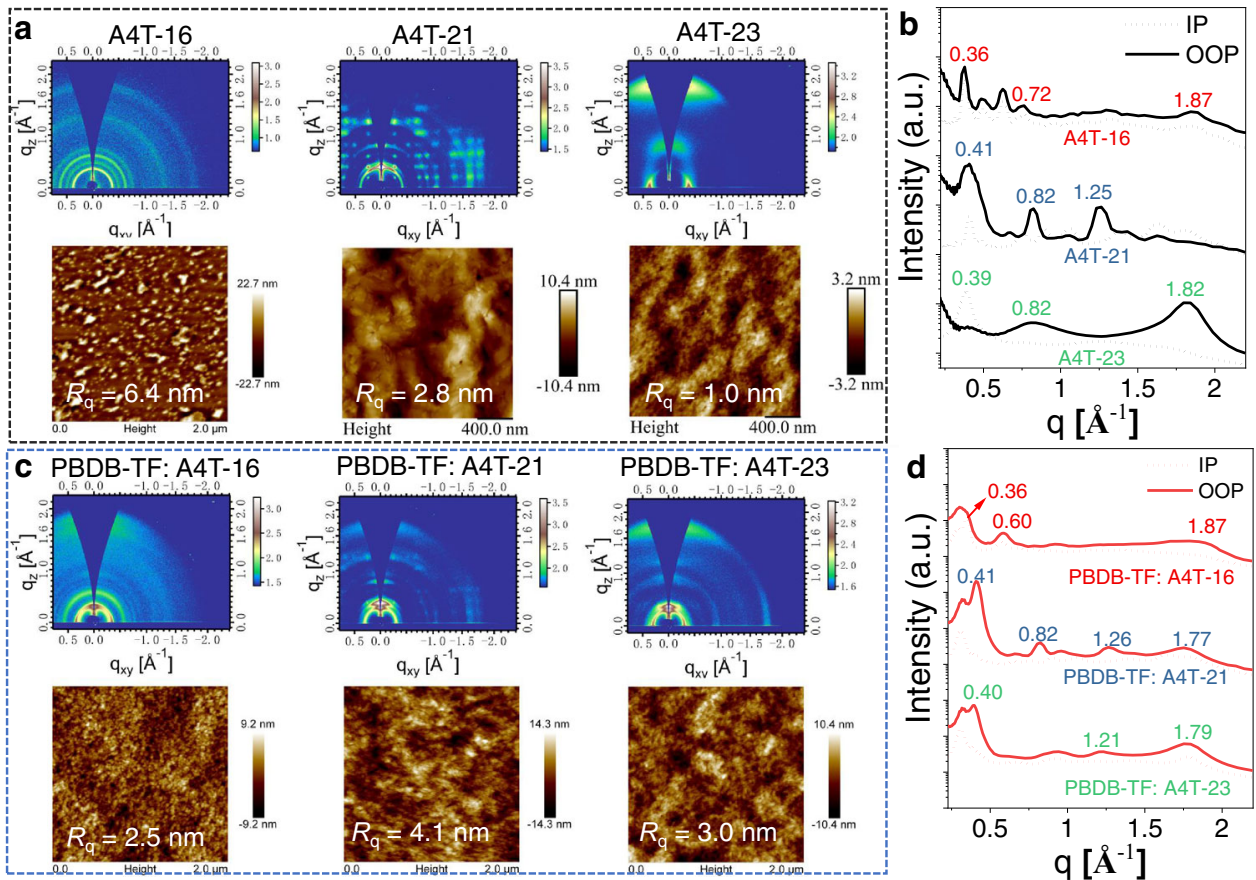

Fig. 6 GIWAXS and AFM analysis of the blend films. a GIWAXS patterns and AFM height images, $\mathbf{b}$ the IP, OOP extracted line-cut profiles of the A4T-16-, A4T-21- A4T-23- pure films, c GIWAXS patterns and AFM height images, $\mathbf{d}$ the IP, OOP extracted line-cut profiles of the PBDB-TF: A4T-16-, PBDB-TF: A4T21-, PBDB-TF: A4T-23- blend films.

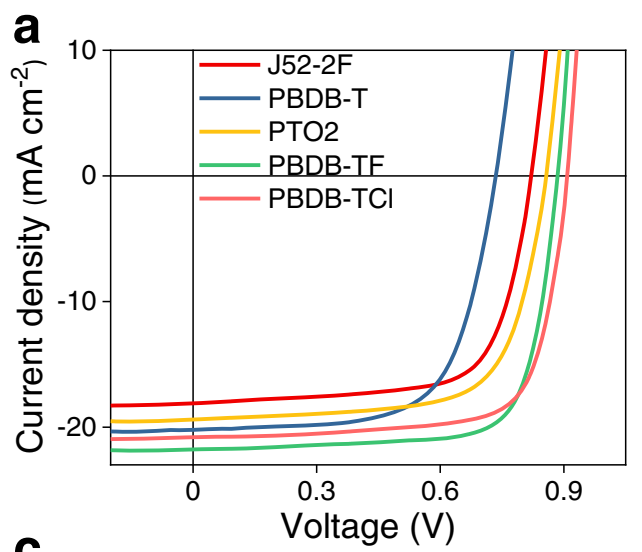

b
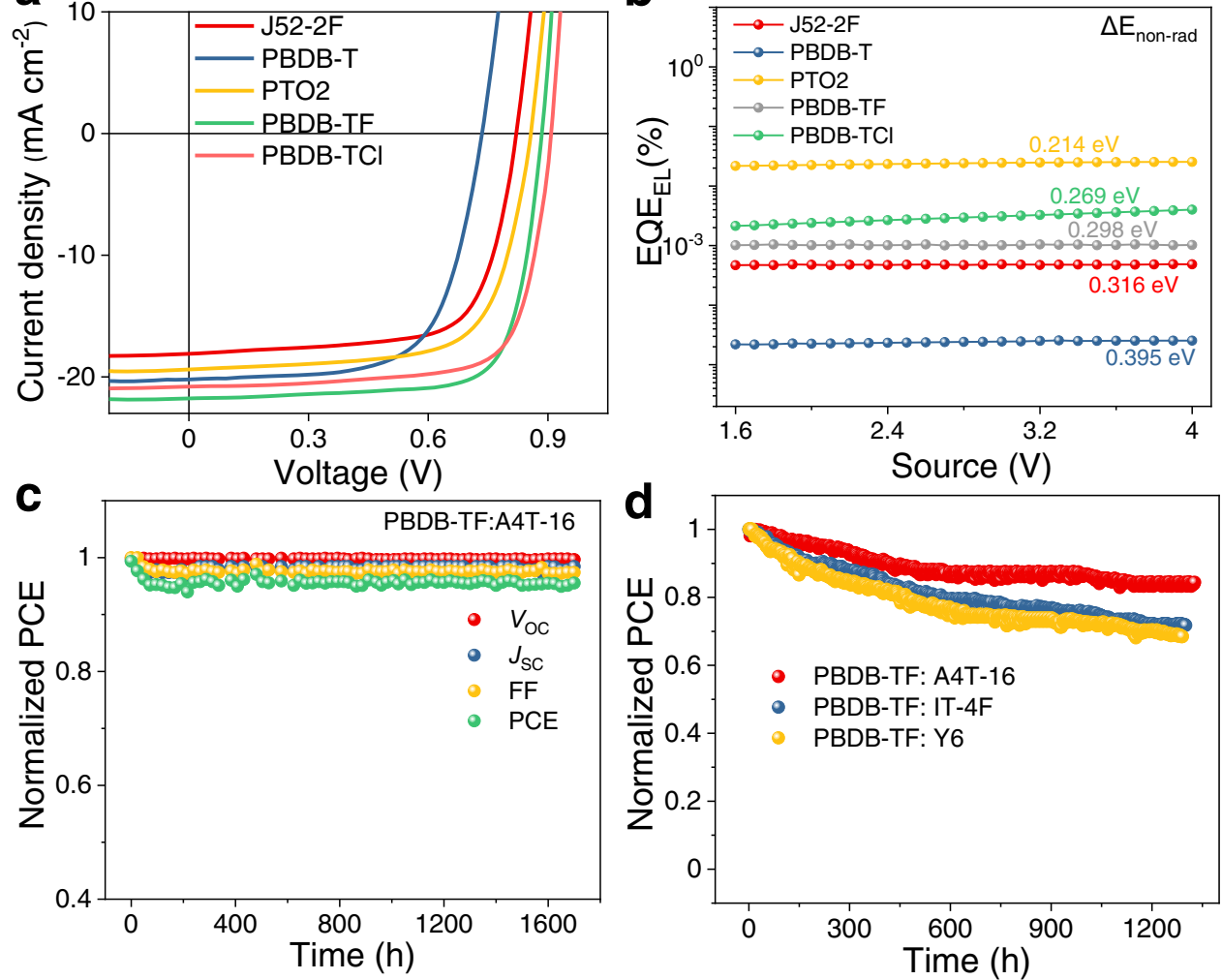

Fig. 7 Universality of A4T-16 and device stability. a $J-V$ curves, b EQE $E_{E L}$ curves of polymer donors: A4T-16-based cells. c The storage stability of the encapsulated PBDB-TF: A4T-16 cell (the encapsulated cells were stored in a glovebox under the nitrogen atmosphere). d Photo-stability of IT-4F-, Y6- and A4T-16-based cells (the encapsulated cells were measured in air under the illumination of AM $1.5 \mathrm{G}, 100 \mathrm{~mW} \mathrm{~cm}{ }^{-2}$ at $\sim 50 \pm 3^{\circ} \mathrm{C}$ ). 
Table 2 Photovoltaic parameters of the polymers: A4T-16-based cells.

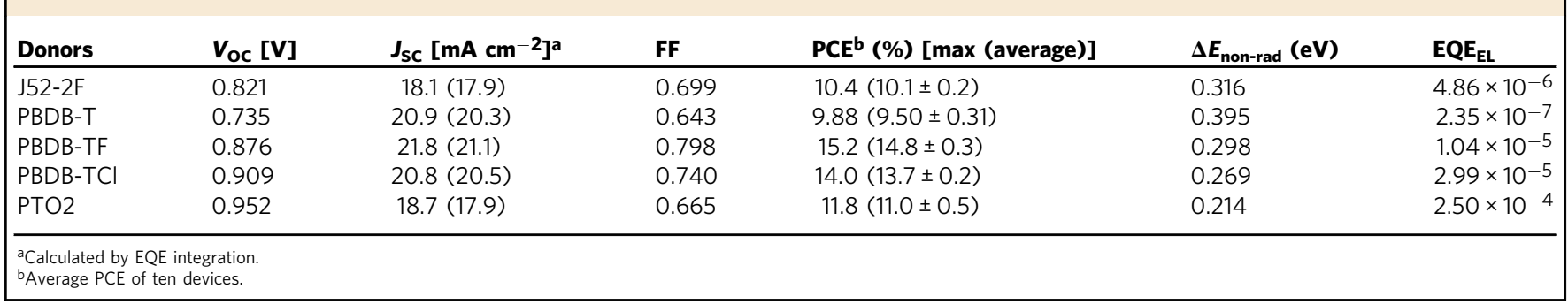

$J_{\text {SCS }}$ and FFs. In addition, for these cells, although the EQE onsets at the long-wavelength direction are the same (Supplementary Fig. 15a), the $V_{\mathrm{OC}}$ values vary from 0.735 to $0.952 \mathrm{~V}$, meaning the $V_{\text {loss }}$ in these devices may be quite different. Therefore, we measured the EL properties and then calculated the $\Delta E_{\text {non-rad }}$ values of the cells. As shown in Fig. $7 \mathrm{~b}$ and Table 2, for the PTO2:A4T-16-based cell, the $\Delta E_{\text {non-rad }}$ value is as low as $0.214 \mathrm{eV}$, which is even smaller than the aforementioned Y6based cell; for the PBDB-T:A4T-16-based cell, this value is as large as $0.395 \mathrm{eV}$, resulting in a low $V_{\mathrm{OC}}$ of $0.735 \mathrm{~V}$. Overall, these results clearly demonstrate that A4T-16 is an excellent non-fused acceptor with good universality and has potential to achieve higher photovoltaic performance.

In addition to high PCE, the long-term device stabilities of OPV cells in storage and under the continuous sunlight illumination are very important to their practical application in the future. Here, we studied the storage stability of the encapsulated PBDB-TF:A4T-16 cell and found that the cell maintains $95 \%$ of its initial PCE after $1700 \mathrm{~h}$ storage under the nitrogen atmosphere with a glovebox temperature of $22 \pm 5^{\circ} \mathrm{C}$. (Fig. 7c). Furthermore, the device stability of the cell under the continuous 1-sun-equivalent illumination was investigated. In order to make clear comparisons, we also tested the stabilities of the PBDB-TF:IT-4F- and PBDB-TF:Y6-based cells in parallel. It is worth noting that this part of stability measurements was carried out under the ambient atmosphere with $40 \pm 10 \%$ humidity, and the encapsulated cells were naturally heated up to $50 \pm 3^{\circ} \mathrm{C}$ by the continuous illumination. As shown in Fig. 7d, the PBDB-TF:A4T16-based cell retains $\sim 84 \%$ of its initial PCE after $1300 \mathrm{~h}$ illumination, which is higher than those of the two control samples, i.e., $72 \%$ for the PBDB-TF:IT- 4 F-based cell and $71 \%$ for the PBDB-TF:Y6-based cell.

To explore the cause for the different stabilities of the three cells, the intrinsic photochemical stabilities of A4T-16, IT-4F, and Y6 were evaluated by exposing the neat films under the continuous illumination by a $365 \mathrm{~nm}$ UV-light and a solar simulator under the ambient atmosphere. The UV-vis absorption spectra of the neat films were monitored and used as the indicator for their photochemical stabilities. As shown in Supplementary Fig. 16, under $365 \mathrm{~nm}$ UV-light, for the absorption spectra of A4T-16, A4T-23, and Y6 films, the absorbances slightly decreased after 8 days. However, for the IT-4F and A4T21 films, the absorption spectrum greatly changed after 8 days of illumination and are completely bleached after $\sim 16$ days. Under continuous 1-sun-equivalent illumination, the absorbance of the IT-4F and A4T-21 films decreased rapidly in the first $3 \mathrm{~h}$, and no absorption can be detected after $17 \mathrm{~h}$, implying the degradation of IT-4F and A4T-21. By contrast, the absorbance of Y6, A4T-23, and A4T-16 decreases slowly, with a low attenuation after $25 \mathrm{~h}$ (Supplementary Fig. 17). Although further studies on the photochemical stability of these acceptors are still needed, the current results already demonstrate the superiority of A4T-16 and Y6 in realizing stable and efficient OPV cells.
In summary, we designed a bithiophene-based building block (TT-P $i$ ) with a unique molecular geometry, based on which a new non-fused NFA with the superior properties for photovoltaic application was prepared. In TT-P $i$, 2,4,6-trimethylphenyl, a functional group with very high and symmetric steric hindrance, was used to lock the two thiophenes into one plane. The DFT calculation results reveal that this new method is not only more effective in enhancing the conformational stability of the planar structure than the broadly used noncovalent conformational lockers like O...S and S...F, but also provide the steric hindrance for inducing the formation of the favourable intermolecular packing mode of the A-D-A-structured NFAs. The single-crystal structure of A4T-16 shows the end-capping groups of the adjacent molecules stack in the perpendicular and horizontal direction with a very compact $\pi-\pi$ distance of $3.35 \AA$, by which a 3D interpenetrated network can be formed. GIWAXS measurements demonstrate that the $3 \mathrm{D}$ network of A4T-16 could be partially retained in the blend film.

The PBDB-TF:A4T-16-based cell not only yields a PCE of $15.2 \%$, the highest value for the cells based on non-fused NFAs, but also exhibits excellent long-term stability under the continuous simulated 1-sun-illumination due to the superior photochemical stability of the A4T-16. Device physics studies indicate the PBDB-TF:A4T-16-based OPV cells have less energetic disorder than the analogues, and by blending with the other polymer donors, the non-radiative energy loss of the A4T-16based cells can be as low as $0.214 \mathrm{eV}$, which is even smaller than that of the Y6-based cell. The photovoltaic characterizations also reveal that A4T-16 has good universality. Overall, the molecular design method and the superior photovoltaic properties of A4T-16 can be used as the guidance for developing new non-fused NFAs with the merits of high efficiency, high stability, and low cost.

Reporting summary. Further information on research design is available in the Nature Research Reporting Summary linked to this article.

\section{Data availability}

The data that support the findings of this study are available from the corresponding author on request. The X-ray crystallographic coordinate for A4T-16 reported in this study has been deposited at the Cambridge Crystallographic Data Centre (CCDC), under deposition numbers 2074697. These data can be obtained free of charge from The Cambridge Crystallographic Data Centre via www.ccdc.cam.ac.uk/data_request/cif.

Received: 8 April 2021; Accepted: 4 August 2021; Published online: 24 August 2021

\section{References}

1. Brabec, C. J. \& Durrant, J. R. Solution-processed organic solar cells. MRS Bull. 33, 670-675 (2008)

2. Arias, A. C., MacKenzie, J. D., McCulloch, I., Rivnay, J. \& Salleo, A. Materials and applications for large area electronics: solution-based approaches. Chem. Rev. 110, 3-24 (2010). 
3. Gaudiana, R. Third-generation photovoltaic technology-the potential for low-cost solar energy conversion. J. Phys. Chem. Lett. 1, 1288-1289 (2010).

4. Lin, Y. Z. et al. An electron acceptor challenging fullerenes for efficient polymer solar cells. Adv. Mater. 27, 1170-1174 (2015).

5. Zhao, W. C. et al. Fullerene-free polymer solar cells with over $11 \%$ efficiency and excellent thermal stability. Adv. Mater. 28, 4734-4739 (2016).

6. Zhao, F. et al. Single-junction binary-blend nonfullerene polymer solar cells with $12.1 \%$ efficiency. Adv. Mater. 29, 1700144 (2017).

7. Yuan, J. et al. Enabling low voltage losses and high photocurrent in fullerenefree organic photovoltaics. Nat. Commun. 10, 570 (2019).

8. Yuan, J. et al. Single-junction organic solar cell with over $15 \%$ efficiency using fused-ring acceptor with electron-deficient core. Joule 3, 1140-1151 (2019).

9. Zhang, Z. et al. Selenium heterocyclic electron acceptor with small Urbach energy for as-cast high-performance organic solar cells. J. Am. Chem. Soc. 142, 18741-18745 (2020).

10. Chen, Y., Zheng, Y., Jiang, Y., Fan, H. \& Zhu, X. Carbon-bridged 1,2-bis(2thienyl)ethylene: an extremely electron rich dithiophene building block enabling electron acceptors with absorption above $1000 \mathrm{~nm}$ for highly sensitive nir photodetectors. J. Am. Chem. Soc. 143, 4281-4289 (2021).

11. Lin, Y. et al. High-performance electron acceptor with thienyl side chains for organic photovoltaics. J. Am. Chem. Soc. 138, 4955-4961 (2016).

12. Yao, H. et al. Design, synthesis, and photovoltaic characterization of a small molecular acceptor with an ultra-narrow band gap.Angew. Chem. Int. Ed. 56, 3045-3049 (2017).

13. Huang, C. et al. Highly efficient organic solar cells based on s,n-heteroacene non-fullerene acceptors. Chem. Mater. 30, 5429-5434 (2018).

14. Ma, Y., Zhang, M., Tang, Y., Ma, W. \& Zheng, Q. Angular-shaped dithienonaphthalene-based nonfullerene acceptor for high-performance polymer solar cells with large open-circuit voltages and minimal energy losses. Chem. Mater. 29, 9775-9785 (2017).

15. Liu, F. et al. Efficient semitransparent solar cells with high nir responsiveness enabled by a small-bandgap electron acceptor. Adv. Mater. 29, 1606574 (2017).

16. Jiang, K. et al. Alkyl chain tuning of small molecule acceptors for efficient organic solar cells. Joule 3, 3020-3033 (2019).

17. Ma, Y. et al. Efficient organic solar cells from molecular orientation control of m-series acceptors. Joule 5, 197-209 (2021).

18. Feng, K. et al. Cyano-functionalized bithiophene imide-based n-type polymer semiconductors: Synthesis, structure-property correlations, and thermoelectric performance. J. Am. Chem. Soc. 143, 1539-1552 (2021).

19. Li, T. et al. Butterfly effects arising from starting materials in fused-ring electron acceptors. J. Am. Chem. Soc. 142, 20124-20133 (2020).

20. Lin, F., Jiang, K., Kaminsky, W., Zhu, Z. \& Jen, A. K. Y. A non-fullerene acceptor with enhanced intermolecular $\pi$-core interaction for highperformance organic solar cells. J. Am. Chem. Soc. 142, 15246-15251 (2020).

21. Min, J. et al. Evaluation of electron donor materials for solution-processed organic solar cells via a novel figure of merit. Adv. Energy Mater. 7, 1700465 (2017).

22. Ma, L., Zhang, S., Wang, J., Xu, Y. \& Hou, J. Recent advances in non-fullerene organic solar cells: From lab to fab. Chem. Commun. 56, 14337-14352 (2020).

23. Kalowekamo, J. \& Baker, E. Estimating the manufacturing cost of purely organic solar cells. Sol. Energy 83, 1224-1231 (2009).

24. Wadsworth, A. et al. Critical review of the molecular design progress in nonfullerene electron acceptors towards commercially viable organic solar cells. Chem. Soc. Rev. 48, 1596-1625 (2019).

25. Hou, J. H., Inganas, O., Friend, R. H. \& Gao, F. Organic solar cells based on non-fullerene acceptors. Nat. Mater. 17, 119-128 (2018).

26. Yan, C. et al. Non-fullerene acceptors for organic solar cells. Nat. Rev. Mater. 3, 18003 (2018).

27. Zhang, G. et al. Nonfullerene acceptor molecules for bulk heterojunction organic solar cells. Chem. Rev. 118, 3447-3507 (2018).

28. Liu, Y. et al. Nonfullerene acceptors with enhanced solubility and ordered packing for high-efficiency polymer solar cells. ACS Energy Lett. 3, 1832-1839 (2018).

29. Lai, H. \& He, F. Crystal engineering in organic photovoltaic acceptors: a $3 \mathrm{~d}$ network approach. Adv. Energy Mater. 10, 2002678 (2020).

30. Aldrich, T. J. et al. Fluorination effects on indacenodithienothiophene acceptor packing and electronic structure, end-group redistribution, and solar cell photovoltaic response. J. Am. Chem. Soc. 141, 3274-3287 (2019).

31. Lai, H. et al. Trifluoromethylation enables a $3 \mathrm{~d}$ interpenetrated low-band-gap acceptor for efficient organic solar cells. Joule 4, 688-700 (2020).

32. Li, X. et al. Synthesis and photovoltaic properties of a simple non-fused small molecule acceptor. Org. Electron. 58, 133-138 (2018).

33. Yu, Z.-P. et al. Simple non-fused electron acceptors for efficient and stable organic solar cells. Nat. Commun. 10, 2152 (2019).
34. Chen, Y.-N. et al. A fully non-fused ring acceptor with planar backbone and near-ir absorption for high performance polymer solar cells. Angew. Chem., Int. Ed. 59, 22714-22720 (2020)

35. Li, Y. et al. Simple non-fullerene electron acceptors with unfused core for organic solar cells. Chin. Chem. Lett. 30, 222-224 (2019).

36. Huang, H. et al. Noncovalently fused-ring electron acceptors with nearinfrared absorption for high-performance organic solar cells. Nat. Commun. 10, 3038 (2019).

37. Zhou, Y. et al. High-efficiency organic solar cells based on a low-cost fully non-fused electron acceptor. Adv. Funct. Mater. 31, 2101742 (2021).

38. Wang, X. et al. Insights into out-of-plane side chains effects on optoelectronic and photovoltaic properties of simple non-fused electron acceptors. Org. Electron. 89, 106029 (2021)

39. Wen, T.-J. et al. Simple non-fused electron acceptors leading to efficient organic photovoltaics. Angew. Chem., Int. Ed. 60, 12964-12970 (2021)

40. Zhan, L. et al. Layer-by-layer processed ternary organic photovoltaics with efficiency over 18\%. Adv. Mater. 33, 2007231 (2021).

41. Zhang, M. et al. Single-layered organic photovoltaics with double cascading charge transport pathways: 18\% efficiencies. Nat. Commun. 12, 309 (2021).

42. Schmidt, A. \& Rahimi, A. A versatile catalyst system for Suzuki-Miyamura syntheses of sterically hindered biaryls employing a cyclobutene-1,2bis(imidazolium) salt. Chem. Commun. 46, 2995 (2010).

43. Organ, M. G., Calimsiz, S., Sayah, M., Hoi, K. H. \& Lough, A. J. Pd-Pepsiipent: an active, sterically demanding cross-coupling catalyst and its application in the synthesis of tetra-ortho-substituted biaryls. Angew. Chem., Int. Ed. 48, 2383-2387 (2009).

44. Wu Yao, D. Z. et al. Yue Guizhou application of mesitylboronic acid and its esters in coupling reactions. Chin. J. Org. Chem. 38, 2896-2926 (2018).

45. Swick, S. et al. Closely packed, low reorganization energy $\pi$-extended postfullerene acceptors for efficient polymer solar cells. Proc. Natl Acad. Sci. 115, 201807535 (2018)

46. Li, W. et al. A high-efficiency organic solar cell enabled by the strong intramolecular electron push-pull effect of the nonfullerene acceptor. Adv. Mater. 30, 1707170 (2018).

47. Cong, Z. et al. Efficient polymer solar cells having high open-circuit voltage and low energy loss enabled by a main-chain twisted small molecular acceptor. ACS Appl. Mater. Interfaces 11, 16795-16803 (2019).

48. $\mathrm{Wu}, \mathrm{Y}$. et al. The crucial role of chlorinated thiophene orientation in conjugated polymers for photovoltaic devices. Angew. Chem., Int. Ed. 57, 12911-12915 (2018).

49. Li, Y. et al. Controlling crystallite orientation of diketopyrrolopyrrole-based small molecules in thin films for highly reproducible multilevel memory device: role of furan substitution. Adv. Funct. Mater. 25, 4246-4254 (2015).

50. Qian, D. et al. Design rules for minimizing voltage losses in high-efficiency organic solar cells. Nat. Mater. 17, 703-709 (2018).

51. Liu, J. et al. Fast charge separation in a non-fullerene organic solar cell with a small driving force. Nat. Energy 1, 16089 (2016).

52. Malliaras, G. G., Salem, J. R., Brock, P. J. \& Scott, C. Electrical characteristics and efficiency of single-layer organic light-emitting diodes. Phys. Rev. B 58, R13411-R13414 (1998)

53. Zhao, W. et al. Molecular optimization enables over $13 \%$ efficiency in organic solar cells. J. Am. Chem. Soc. 139, 7148-7151 (2017).

54. Limpinsel, M., Wagenpfahl, A., Mingebach, M., Deibel, C. \& Dyakonov, V. Photocurrent in bulk heterojunction solar cells. Phys. Rev. B 81, 085203 (2010).

55. Cowan, S. R., Roy, A. \& Heeger, A. J. Recombination in polymer-fullerene bulk heterojunction solar cells. Phys. Rev. B 82, 245207 (2010).

56. Cui, Y. et al. Single-junction organic photovoltaic cells with approaching $18 \%$ efficiency. Adv. Mater. 32, 1908205 (2020).

57. Cui, Y. et al. Over $16 \%$ efficiency organic photovoltaic cells enabled by a chlorinated acceptor with increased open-circuit voltages. Nat. Commun. 10, 2515 (2019)

58. Ye, L. et al. Quantitative relations between interaction parameter, miscibility and function in organic solar cells. Nat. Mater. 17, 253-260 (2018).

59. Liang, Z. et al. Optimization requirements of efficient polythiophene: nonfullerene organic solar cells. Joule 4, 1278-1295 (2020).

60. Ran, N. A. et al. Harvesting the full potential of photons with organic solar cells. Adv. Mater. 28, 1482-1488 (2016).

61. An, N. et al. Solution-processed organic solar cells with high open-circuit voltage of $1.3 \mathrm{~V}$ and low non-radiative voltage loss of $0.16 \mathrm{~V}$. Adv. Mater. 32, $2002122(2020)$

62. $\mathrm{Xu}, \mathrm{Y}$. et al. Tuning the hybridization of local exciton and charge-transfer states in highly efficient organic photovoltaic cells. Angew. Chem., Int. Ed. 59, 9004-9010 (2020)

63. Eisner, F. D. et al. Hybridization of local exciton and charge-transfer states reduces nonradiative voltage losses in organic solar cells. J. Am. Chem. Soc. 141, 6362-6374 (2019). 


\section{Acknowledgements}

This work was supported by the National Key Research and Development Programme of China (2019YFE0116700), and the Basic and Applied Basic Research Major Programme of Guangdong Province (2019B030302007). The authors would like to acknowledge the support from the National Natural Science Foundation of China (NSFC, 21835006, 91633301, 51961135103, 21734008, and 51673201). Beijing National Laboratory for Molecular Sciences (BNLMS-CXXM-201903).

\section{Author contributions}

S.Z. and J.H. conceived the idea. L.M. performed the fabrication/test of the OPV cells. J.Zhu and J.R. synthesized these acceptors, J.W. conducted the theoretical calculations and carried out the absorption and energy level measurements. J.R. provided TEM images. J.Z. conducted the GIWAXS measurements. L.M., S.Z. and J.H. prepared the paper. All authors commented on the paper. J.H. supervised the project.

\section{Competing interests}

The authors declare no competing interests.

\section{Additional information}

Supplementary information The online version contains supplementary material available at https://doi.org/10.1038/s41467-021-25394-w.

Correspondence and requests for materials should be addressed to S.Z. or J.H.
Peer review information Nature Communications thanks the anonymous reviewer(s) for their contribution to the peer review of this work

Reprints and permission information is available at http://www.nature.com/reprints

Publisher's note Springer Nature remains neutral with regard to jurisdictional claims in published maps and institutional affiliations.

(c) The Author(s) 2021 